\title{
Profiling of Growth and Yield Parameters of Eggplant as Influenced by the Cropping Season
}

\author{
A.V.V. Koundinya ${ }^{1,2} *$, A. Das ${ }^{1}$, P. Pradeep Kumar ${ }^{1}$ and M.K. Pandit ${ }^{1}$ \\ ${ }^{\mathbf{1}}$ Department of Vegetable Crops, Bidhan Chandra Krishi Viswavidyalaya, \\ Mohanpur, West-Bengal, India \\ ${ }^{2}$ ICAR-Central Tuber Crops Research Institute, Thiruvananthapuram, Kerala, India \\ *Corresponding author
}

\begin{tabular}{|c|c|}
\hline & A B S T R A C T \\
\hline $\begin{array}{l}\text { Season, Genotype, } \\
\text { Growth, Yield, } \\
\text { Brinjal. }\end{array}$ & \multirow{3}{*}{$\begin{array}{l}\text { The present study was carried out at AB District Seed Farm, BCKV, Kalyani Simanta, } \\
\text { West Bengal, India during spring-summer 2012-13 and 2013-14, autumn-winter 2013-14 } \\
\text { and 2014-15 with } 40 \text { brinjal genotypes in Randomized Block Design with two replications. } \\
\text { Highly significant differences were observed for majority of the characters for the two } \\
\text { factors viz., genotypes }(\mathrm{G}) \text { and season (S) and their interaction (G X S). Paired t-test also } \\
\text { clearly demonstrated the influence of season on the performance of eggplant germplasm. } \\
\text { The vegetative phage was favoured and earlier flowering took place during spring summer } \\
\text { and the reproductive phage was favoured during autumn winter. The characters plant } \\
\text { height and primary branches per plant were high and the genotypes took less number of } \\
\text { days for flowering during spring summer. Other important yield components like number } \\
\text { of fruits per plant, fruit weight, fruit yield per plant and harvesting index were high during } \\
\text { autumn winter. }\end{array}$} \\
\hline Article Info & \\
\hline $\begin{array}{l}\text { Accepted: } \\
04 \text { April } 2017 \\
\text { Available Online: } \\
10 \text { May } 2017\end{array}$ & \\
\hline
\end{tabular}

\section{Introduction}

Eggplant or brinjal (Solanum melongena L.) is the major vegetable grown and consumed in India. The fully grown tender fruits are widely used in various culinary preparations viz., sliced baji, stuffed curry, bartha, chutni and pickle. It is rich in vitamins like thiamine, niacin, pantothenic acid and folacin as well as essential minerals like calcium $(\mathrm{Ca})$, iron $(\mathrm{Fe})$, potassium $(\mathrm{K})$, and zinc $(\mathrm{Zn})$, copper $(\mathrm{Cu})$ and manganese $(\mathrm{Mn})$, chromium $(\mathrm{Cr})$ and selenium (Se) (Kowalski et al., 2003). Eggplant is grown throughout the year in the lower Gangetic alluvial plains of West Bengal. Eggplant is a thermo-sensitive crop and cultivars of eggplant not only differ genetically in a vast number of properties, namely, plant height, earliness, length of fruiting period, number of fruits and yielding ability but also differ over their places of cultivation and seasons. Therefore, characterization of available germplasm in different seasons provides deep insight into the environmental interference in the complete expression of the genetic worth of the plants. Hence, the present study was undertaken with a view to provide deep insight into the seasonal differences in the plant growth, flowering and yield. 


\section{Materials and Methods}

The present study was carried out in the $\mathrm{AB}$ District Seed Farm, BCKV, Kalyani Simanta (Latitude $22^{0} 58^{\prime} \mathrm{N}$ and Longitude $88^{0} 32^{\prime} \mathrm{E}$ ), West Bengal, India during spring-summer (February-June) 2012-13 and 2013-14 and autumn-winter (September-March) 2013-14 and 2014-15. The study site is flat and is located at an altitude of $9.75 \mathrm{~m}$ above mean sea level. The experimental material was comprised of 40 eggplant germplasm, including local cultivars of West Bengal and varieties \& breeding lines obtained from other parts of the country. The experiment was laid out in a Randomized Block Design (RBD) with two replications. In each replication, each genotype was grown on a plot of $3 \mathrm{X}$ $2.25 \mathrm{~m}$ size, accommodating 12 plants with the row-to-row spacing of $75 \mathrm{~cm}$ and plant-toplant spacing of $75 \mathrm{~cm}$. The seeds were sown in raised nursery bed and the seedlings were transplanted to the main field when they were four weeks old. The recommended package of practices was followed to maintain a good crop stand. Observations were taken on growth parameters (plant height, number of primary branches per plant), earliness indicators (days to $1^{\text {st }}$ and 50\% flowering) and yield traits (number of fruits per plant, fruit weight, fruit yield per plant, harvest index) in each season. The analysis of variance in two factorial RBD fashion for different characters was carried out, in order to assess the variability among the genotypes and across the seasons as given by Cochran \& Cox (1957). Paired t-test was done as per McDonald (2014) to test the null hypothesis $\left(\mathrm{H}_{0}=\right.$ No significant differences were observed between the two seasons in terms of eggplant performance) against the alternate hypothesis $\left(\mathrm{H}_{1}=\right.$ the performance of eggplant in both the seasons was significantly different) in order to confirm the thermosensitive nature of the eggplant. The software used for the calculation was MINITAB v. 16.

\section{Results and Discussion}

The data collected pertaining to various morphological and yield components in both spring-summer and autumn-winter seasons were subjected to ANOVA of two Factorial Randomized Block Design. Highly significant differences were observed for plant height, days to first flowering, days to $50 \%$ flowering, number of fruits per plant, fruit yield per plant and harvest index for the two factors viz., genotypes (G) and season (S) and their interaction $(\mathrm{GX} \mathrm{S})$, which indicated the presence of sufficient amount of variability in the population and their differential performance in both the seasons in the lower Gangetic alluvial plains of West-Bengal. The characters, numbers of primary branches per plant and fruit weight, were found to have significant difference among the genotypes for the two factors individually but, not for the interaction of these two factors (Table 1). Similar results were earlier reported by Pandit et al., (2010) in the same region. The characters plant height, primary branches per plant were high and the genotypes took less number of days for flowering during springsummer. Other important yield components like number of fruits per plant, fruit weight, fruit yield per plant and harvesting index were high during autumn-winter.

Paired t-test was done to see whether the seasonal differences are statistically significant or not. It takes the difference of the performance of each genotype in both the seasons into account. Therefore, it is more effective than ANOVA in understanding the seasonal differences statistically. The paired $t-$ calculated values for all the characters were present in table 1 . They were compared with the t-table value at 38 degrees of freedom and found that there was significant difference in the performance of genotypes in both the seasons. This confirms the thermo-sensitive nature of the eggplant. 
The frequencies of the men differences (spring-summer-autumn-winter) were presented in figure 1. These histograms of paired t-test (Fig. 1) illustrated that all the 40 eggplant genotypes differed in both the seasons for plant height, days to $1^{\text {st }}$ flowering, days to $50 \%$ flowering, fruit yield per plant and harvesting index as none of the genotype had similar performance for these traits.

Highly 12 genotypes for number of fruits per plant and 3 genotypes for fruit weight had similar performance in both the seasons.

However, in all the cases the null hypothesis $\left(\mathrm{H}_{0}: X-Y=0\right.$; where $\mathrm{X}$ and $\mathrm{Y}$ are the means of spring-summer and autumn-winter) is out of the confidence interval of the population mean differences, thus, specifies the acceptance of alternate hypothesis $\left(\mathrm{H}_{1}: X-Y \neq 0\right)$ for all the growth, earliness and yield parameters.
Figure 1 also demonstrated that all the genotypes responded positively for plant height and number of primary branches per plant during spring-summer and the reduction in height and primary branches per plant was noticed in all the genotypes during autumnwinter. Only one genotype took fewer days to first and 50\% flowering (KS-8329), while remaining all flowered lately during autumnwinter. During spring-summer, two genotypes (KS-8329 and KS-8103) had more number of fruits while fruit weight, fruit yield per plant and harvesting index were high for only one genotype (KS-8103).

The mean performance of genotypes in both the seasons for various morphological and yield parameters are presented in table 2 . Plant height ranged from $79.8 \mathrm{~cm}$ (Sada Makra) to $140.1 \mathrm{~cm}$ (KS-8329) with a mean value of $106.4 \mathrm{~cm}$ during spring-summer and $54.1 \mathrm{~cm}(\mathrm{H}-8)$ to $95.5 \mathrm{~cm}$ (KS-9010) with a mean value of $72.3 \mathrm{~cm}$ during autumn-winter.

Table.1 ANOVA of two factorial RBD and Paired t-test of various characters

\begin{tabular}{|c|c|c|c|c|c|c|c|c|c|}
\hline & & 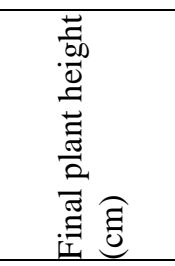 & 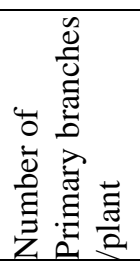 & 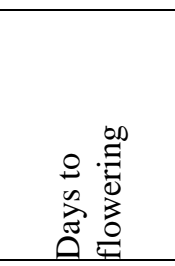 & 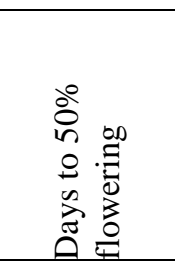 & 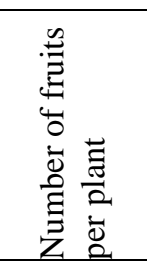 & 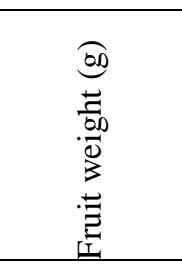 & 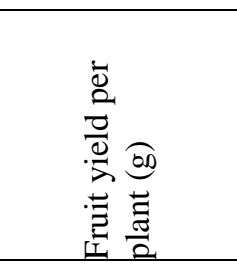 & 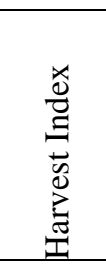 \\
\hline $\begin{array}{l}\text { Source of } \\
\text { Variation }\end{array}$ & DF & \multicolumn{8}{|c|}{ Mean Sum of Squares } \\
\hline Season & 1 & $46,343.84 *$ & $83.457 *$ & $15,441.53^{*}$ & $18,949.81^{*}$ & $708.377 *$ & $17,362.15^{*}$ & $12,842,665.41 *$ & $1.454 *$ \\
\hline Genotype & 39 & $762.265^{*}$ & $1.337 *$ & $195.022 *$ & $167.491 *$ & $288.63^{*}$ & $9,230.12 *$ & $848,118.16^{*}$ & $0.074 *$ \\
\hline$S \times G$ & 39 & $202.258 *$ & 0.382 & $69.816^{*}$ & $67.252 *$ & $26.379 *$ & 228.819 & $207,971.66^{*}$ & $0.008^{*}$ \\
\hline Error & 79 & 47.437 & 0.618 & 17.207 & 23.006 & 4.331 & 300.444 & $45,081.72$ & 0.005 \\
\hline \multicolumn{2}{|l|}{ Parameter } & \multicolumn{8}{|c|}{ Paired t-test (d.f.=38) } \\
\hline \multicolumn{2}{|c|}{ Spring-summer } & 106.4 & 7.1 & 56.0 & 61.3 & 8.9 & 101.4 & 680.0 & 0.43 \\
\hline \multicolumn{2}{|c|}{ Autumn-winter } & 72.3 & 5.7 & 75.7 & 83.0 & 13.1 & 122.2 & 1246.7 & 0.62 \\
\hline \multicolumn{2}{|c|}{$\begin{array}{l}\text { Paired t- } \\
\text { calculated }\end{array}$} & $15.14^{*}$ & $14.61^{*}$ & $14.87^{*}$ & $16.80^{*}$ & $5.18^{*}$ & $8.71 *$ & $7.86^{*}$ & $13.97^{*}$ \\
\hline
\end{tabular}

*Significant at $5 \%$ level of significance 
Table.2 Mean performance of 40 brinjal germplasm for growth and earliness traits in two seasons

\begin{tabular}{|c|c|c|c|c|c|c|c|c|c|c|c|c|c|}
\hline \multirow{2}{*}{ S.No } & \multirow{2}{*}{ Genotypes } & \multicolumn{3}{|c|}{ Final plant height $(\mathrm{cm})$} & \multicolumn{3}{|c|}{ Number of Primary branches per plant } & \multicolumn{3}{|c|}{ Days to first flowering } & \multicolumn{3}{|c|}{ Days to $50 \%$ flowering } \\
\hline & & SS & $\mathrm{AW}$ & Mean & SS & $\mathrm{AW}$ & Mean & SS & AW & Mean & SS & $\mathrm{AW}$ & Mean \\
\hline 1 & Lamba Kuli & 84.7 & 61.2 & 72.9 & 6.6 & 5.7 & 6.2 & 56.0 & 74.5 & 65.3 & 62.0 & 80.3 & 71.2 \\
\hline 2 & Muktamala & 88.8 & 63.6 & 76.2 & 7.7 & 6.5 & 7.1 & 58.2 & 82.1 & 70.1 & 64.3 & 90.9 & 77.6 \\
\hline 3 & Samrat & 89.2 & 70.7 & 79.9 & 7.3 & 6.9 & 7.1 & 61.4 & 76.6 & 69.0 & 68.5 & 81.3 & 74.9 \\
\hline 4 & Makra & 113.2 & 70.3 & 91.7 & 6.3 & 5.4 & 5.9 & 41.6 & 74.6 & 58.1 & 46.0 & 85.0 & 65.5 \\
\hline 5 & Makra Midlong & 135.5 & 75.3 & 105.4 & 8.2 & 7.0 & 7.6 & 49.5 & 75.3 & 62.4 & 56.3 & 85.5 & 70.9 \\
\hline 6 & Kalo Makra & 124.7 & 86.2 & 105.5 & 8.3 & 6.1 & 7.2 & 42.5 & 68.4 & 55.5 & 45.8 & 79.6 & 62.7 \\
\hline 7 & Lal Lamba & 129.7 & 89.3 & 109.5 & 7.8 & 6.5 & 7.2 & 39.7 & 48.3 & 44.0 & 42.3 & 55.1 & 48.7 \\
\hline 8 & $B C B-123$ & 136.3 & 82.0 & 109.2 & 7.7 & 5.7 & 6.7 & 43.3 & 47.5 & 45.4 & 47.0 & 57.0 & 52.0 \\
\hline 9 & Muktakeshi Lal & 100.6 & 71.2 & 85.9 & 7.7 & 4.6 & 6.1 & 54.1 & 78.3 & 66.2 & 64.0 & 87.0 & 75.5 \\
\hline 10 & Gol Makra & 128.2 & 69.2 & 98.7 & 7.3 & 5.3 & 6.3 & 62.1 & 77.5 & 69.8 & 66.5 & 86.7 & 76.6 \\
\hline 11 & Muktakeshi & 120.4 & 59.3 & 89.9 & 7.0 & 5.8 & 6.4 & 53.9 & 69.6 & 61.8 & 61.0 & 80.6 & 70.8 \\
\hline 12 & Panna & 124.1 & 69.5 & 96.8 & 7.4 & 4.8 & 6.1 & 51.4 & 76.4 & 63.9 & 56.0 & 83.8 & 69.9 \\
\hline 13 & Lal Kuli & 113.2 & 74.3 & 93.8 & 7.8 & 6.3 & 7.0 & 49.7 & 80.8 & 65.3 & 59.3 & 87.7 & 73.5 \\
\hline 14 & Mala & 88.4 & 71.3 & 79.9 & 8.2 & 6.8 & 7.5 & 57.4 & 83.0 & 70.2 & 61.3 & 89.0 & 75.1 \\
\hline 15 & Muktajhuri & 104.5 & 69.2 & 86.8 & 7.7 & 6.1 & 6.9 & 57.3 & 79.4 & 68.4 & 63.3 & 87.1 & 75.2 \\
\hline 16 & Heera & 86.7 & 63.6 & 75.1 & 7.0 & 4.8 & 5.9 & 59.1 & 82.7 & 70.9 & 64.0 & 86.3 & 75.1 \\
\hline 17 & Sada Makra & 79.8 & 61.6 & 70.7 & 6.7 & 6.0 & 6.3 & 57.4 & 82.3 & 69.9 & 61.0 & 88.5 & 74.8 \\
\hline 18 & Nandini & 86.3 & 54.3 & 70.3 & 6.0 & 5.3 & 5.6 & 63.4 & 83.5 & 73.4 & 66.0 & 88.0 & 77.0 \\
\hline 19 & BCB-320 & 94.8 & 67.2 & 81.0 & 6.8 & 5.5 & 6.1 & 59.3 & 81.1 & 70.2 & 65.5 & 87.3 & 76.4 \\
\hline 20 & KS-8407 & 93.1 & 71.3 & 82.2 & 8.0 & 6.8 & 7.4 & 50.1 & 71.6 & 60.8 & 57.3 & 83.3 & 70.3 \\
\hline 21 & KS-8102 & 95.8 & 64.3 & 80.0 & 7.0 & 6.0 & 6.5 & 56.8 & 80.9 & 68.9 & 63.8 & 87.5 & 75.6 \\
\hline 22 & KS-7848 & 87.2 & 62.8 & 75.0 & 7.5 & 4.8 & 6.1 & 59.6 & 86.3 & 72.9 & 64.5 & 89.3 & 76.9 \\
\hline 23 & KS-8317 & 94.6 & 70.3 & 82.4 & 6.7 & 6.3 & 6.5 & 58.9 & 77.4 & 68.1 & 63.0 & 88.9 & 75.9 \\
\hline 24 & Kerala collection-1 & 94.2 & 57.4 & 75.8 & 6.1 & 5.6 & 5.8 & 58.6 & 69.1 & 63.9 & 66.5 & 82.7 & 74.6 \\
\hline 25 & KS-7812 & 89.3 & 64.1 & 76.7 & 6.4 & 5.0 & 5.7 & 55.4 & 84.4 & 69.9 & 63.5 & 87.6 & 75.6 \\
\hline 26 & KS-2011-1 & 98.6 & 84.5 & 91.6 & 6.2 & 5.4 & 5.8 & 58.9 & 88.3 & 73.6 & 62.5 & 92.7 & 77.6 \\
\hline 27 & $\mathrm{H}-8$ & 97.4 & 54.1 & 75.8 & 7.0 & 5.3 & 6.2 & 58.2 & 85.0 & 71.6 & 61.8 & 89.0 & 75.4 \\
\hline 28 & KS-5144 & 97.1 & 63.8 & 80.5 & 6.8 & 5.2 & 6.0 & 53.0 & 82.9 & 67.9 & 61.0 & 88.7 & 74.8 \\
\hline 29 & BR-112 & 87.2 & 55.3 & 71.2 & 7.0 & 5.6 & 6.3 & 60.0 & 70.6 & 65.3 & 64.0 & 76.9 & 70.5 \\
\hline 30 & KS-6308 & 121.6 & 82.3 & 102.0 & 6.8 & 5.3 & 6.0 & 63.0 & 78.1 & 70.6 & 68.3 & 83.9 & 76.1 \\
\hline 31 & KS-8329 & 140.1 & 84.9 & 112.5 & 6.9 & 5.5 & 6.2 & 54.8 & 44.7 & 49.8 & 64.0 & 52.7 & 58.3 \\
\hline 32 & KS-9503 & 101.7 & 90.9 & 96.3 & 7.5 & 6.0 & 6.7 & 54.3 & 70.7 & 62.5 & 61.5 & 80.1 & 70.8 \\
\hline 33 & KS-8805 & 120.7 & 85.9 & 103.3 & 7.4 & 5.2 & 6.3 & 62.0 & 74.3 & 68.1 & 66.5 & 80.5 & 73.5 \\
\hline 34 & KS-8105 & 132.1 & 94.0 & 113.0 & 7.6 & 5.3 & 6.4 & 62.7 & 74.0 & 68.3 & 65.5 & 80.9 & 73.2 \\
\hline 35 & KS-9010 & 110.8 & 95.5 & 103.2 & 7.6 & 6.1 & 6.8 & 58.0 & 73.1 & 65.5 & 60.3 & 82.0 & 71.1 \\
\hline 36 & KS-9504 & 103.4 & 93.9 & 98.6 & 7.1 & 6.2 & 6.6 & 60.3 & 72.4 & 66.4 & 63.5 & 81.6 & 72.6 \\
\hline 37 & KS-8103 & 133.4 & 89.2 & 111.3 & 7.5 & 6.1 & 6.8 & 59.0 & 74.4 & 66.7 & 61.5 & 81.0 & 71.2 \\
\hline 38 & KS-9501 & 136.3 & 81.5 & 108.9 & 6.0 & 4.7 & 5.4 & 59.8 & 78.2 & 69.0 & 64.5 & 84.6 & 74.5 \\
\hline 39 & Local collection-1 & 106.2 & 58.7 & 82.5 & 7.3 & 5.5 & 6.4 & 63.2 & 85.3 & 74.2 & 66.0 & 90.0 & 78.0 \\
\hline 40 & Mukta Hasi & 84.8 & 59.1 & 72.0 & 5.5 & 4.6 & 5.1 & 57.8 & 84.1 & 71.0 & 61.5 & 90.7 & 76.1 \\
\hline \multirow{2}{*}{\multicolumn{2}{|c|}{ Mean }} & 106.4 & 72.3 & & 7.1 & 5.7 & & 56.0 & 75.7 & & 61.3 & 83.0 & \\
\hline & & $\mathrm{S}$ & $\mathrm{G}$ & GX S & $\mathrm{S}$ & $\mathrm{G}$ & GX S & $\mathrm{S}$ & $\mathrm{G}$ & GX S & $\mathrm{S}$ & $\mathrm{G}$ & GXS \\
\hline & S.E (m) & 0.8 & 3.4 & 4.9 & 0.1 & 0.4 & 0.6 & 0.5 & 2.1 & 2.9 & 0.5 & 2.4 & 3.4 \\
\hline & C.D. & 2.2 & 9.7 & 13.7 & 0.2 & 1.1 & N/A & 1.3 & 5.8 & 8.3 & 1.5 & 6.8 & 9.6 \\
\hline
\end{tabular}


Table.3 Mean performance of 40 brinjal germplasm for yield parameters in two seasons

\begin{tabular}{|c|c|c|c|c|c|c|c|c|c|c|c|c|c|}
\hline \multirow{2}{*}{ S.N } & \multirow{2}{*}{ Genotypes } & \multicolumn{3}{|c|}{ Number of fruits per plant } & \multicolumn{3}{|c|}{ Fruit weight $(\mathrm{g})$} & \multicolumn{3}{|c|}{ Fruit yield per plant $(\mathrm{g})$} & \multicolumn{3}{|c|}{ Harvesting Index } \\
\hline & & SS & AW & Mean & SS & $\mathrm{AW}$ & Mean & SS & AW & Mean & SS & AW & Mean \\
\hline 1 & Lamba Kuli & 14.6 & 25.3 & 20.0 & 67.3 & 69.9 & 68.6 & 981.8 & 1775.5 & 1378.7 & 0.69 & 0.83 & 0.76 \\
\hline 2 & Muktamala & 25.5 & 28.5 & 27.0 & 48.3 & 70.7 & 59.5 & 1239.6 & 2032.7 & 1636.1 & 0.69 & 0.85 & 0.77 \\
\hline 3 & Samrat & 30.4 & 36.4 & 33.4 & 42.7 & 51.9 & 47.3 & 1312.4 & 1895.9 & 1604.2 & 0.70 & 0.81 & 0.75 \\
\hline 4 & Makra & 9.2 & 20.5 & 14.8 & 96.3 & 127.5 & 111.9 & 867.2 & 2273.9 & 1570.6 & 0.44 & 0.76 & 0.60 \\
\hline 5 & Makra Midlong & 10.4 & 18.0 & 14.2 & 88.9 & 114.3 & 101.6 & 933.4 & 2025.2 & 1479.3 & 0.56 & 0.78 & 0.67 \\
\hline 6 & Kalo Makra & 5.9 & 11.8 & 8.9 & 190.4 & 204.7 & 197.5 & 1176.5 & 2410.8 & 1793.6 & 0.53 & 0.75 & 0.64 \\
\hline 7 & Lal Lamba & 9.4 & 19.7 & 14.6 & 105.3 & 127.9 & 116.6 & 988.9 & 2575.8 & 1782.3 & 0.48 & 0.78 & 0.63 \\
\hline 8 & $B C B-123$ & 9.6 & 17.8 & 13.7 & 87.7 & 121.0 & 104.3 & 859.1 & 2146.0 & 1502.6 & 0.56 & 0.81 & 0.68 \\
\hline 9 & Muktakeshi Lal & 4.4 & 17.6 & 11.0 & 105.8 & 139.9 & 122.8 & 465.8 & 1628.2 & 1047.0 & 0.37 & 0.69 & 0.53 \\
\hline 10 & Gol Makra & 3.1 & 14.7 & 8.9 & 162.8 & 188.1 & 175.5 & 519.7 & 1916.7 & 1218.2 & 0.31 & 0.67 & 0.49 \\
\hline 11 & Muktakeshi & 5.5 & 7.2 & 6.3 & 97.6 & 109.8 & 103.7 & 542.9 & 779.4 & 661.1 & 0.51 & 0.63 & 0.57 \\
\hline 12 & Panna & 17.4 & 26.7 & 22.0 & 78.8 & 95.2 & 87.0 & 1392.6 & 2429.0 & 1910.8 & 0.60 & 0.80 & 0.70 \\
\hline 13 & Lal Kuli & 21.7 & 29.4 & 25.6 & 51.0 & 57.8 & 54.4 & 1078.6 & 1695.2 & 1386.9 & 0.57 & 0.73 & 0.65 \\
\hline 14 & Mala & 18.4 & 30.7 & 24.5 & 58.2 & 55.7 & 57.0 & 1033.9 & 1712.1 & 1373.0 & 0.66 & 0.79 & 0.72 \\
\hline 15 & Muktajhuri & 28.4 & 36.9 & 32.6 & 33.3 & 49.4 & 41.3 & 950.1 & 1811.1 & 1380.6 & 0.64 & 0.79 & 0.71 \\
\hline 16 & Heera & 15.1 & 26.0 & 20.6 & 52.9 & 63.9 & 58.4 & 799.1 & 1661.7 & 1230.4 & 0.56 & 0.80 & 0.68 \\
\hline 17 & Sada Makra & 5.9 & 6.8 & 6.3 & 106.1 & 127.5 & 116.8 & 625.4 & 878.6 & 752.0 & 0.59 & 0.71 & 0.65 \\
\hline 18 & Nandini & 3.2 & 4.9 & 4.1 & 106.1 & 119.9 & 113.0 & 334.9 & 590.3 & 462.6 & 0.29 & 0.47 & 0.38 \\
\hline 19 & BCB-320 & 7.1 & 6.7 & 6.9 & 89.6 & 127.0 & 108.3 & 620.8 & 840.1 & 730.5 & 0.38 & 0.53 & 0.45 \\
\hline 20 & KS-8407 & 2.9 & 5.2 & 4.0 & 78.8 & 106.4 & 92.6 & 224.1 & 555.7 & 389.9 & 0.24 & 0.55 & 0.40 \\
\hline 21 & KS-8102 & 2.5 & 6.7 & 4.6 & 81.1 & 68.9 & 75.0 & 215.6 & 451.5 & 333.6 & 0.29 & 0.54 & 0.42 \\
\hline 22 & KS-7848 & 2.4 & 5.7 & 4.1 & 87.1 & 89.5 & 88.3 & 212.5 & 503.5 & 358.0 & 0.24 & 0.46 & 0.35 \\
\hline 23 & KS-8317 & 3.6 & 14.5 & 9.1 & 79.4 & 85.0 & 82.2 & 280.8 & 1238.1 & 759.5 & 0.36 & 0.74 & 0.55 \\
\hline 24 & Kerala collection-1 & 8.5 & 15.0 & 11.8 & 68.1 & 101.1 & 84.6 & 580.5 & 1532.5 & 1056.5 & 0.46 & 0.73 & 0.60 \\
\hline 25 & KS-7812 & 3.6 & 4.2 & 3.9 & 99.5 & 136.8 & 118.1 & 367.9 & 586.3 & 477.1 & 0.36 & 0.48 & 0.42 \\
\hline 26 & KS-2011-1 & 2.1 & 2.9 & 2.5 & 101.0 & 160.0 & 130.5 & 196.9 & 459.0 & 327.9 & 0.26 & 0.48 & 0.37 \\
\hline 27 & H-8 & 3.9 & 3.0 & 3.4 & 164.4 & 234.6 & 199.5 & 633.0 & 769.4 & 701.2 & 0.46 & 0.60 & 0.53 \\
\hline 28 & KS-5144 & 3.8 & 5.4 & 4.6 & 92.0 & 122.5 & 107.2 & 349.5 & 662.5 & 506.0 & 0.29 & 0.51 & 0.40 \\
\hline 29 & BR-112 & 4.4 & 5.1 & 4.8 & 128.3 & 148.1 & 138.2 & 563.0 & 763.0 & 663.0 & 0.32 & 0.43 & 0.37 \\
\hline 30 & KS-6308 & 4.1 & 6.0 & 5.1 & 104.8 & 119.5 & 112.1 & 431.8 & 701.1 & 566.5 & 0.27 & 0.47 & 0.37 \\
\hline 31 & KS-8329 & 12.6 & 10.9 & 11.8 & 42.5 & 57.5 & 50.0 & 481.3 & 634.9 & 558.1 & 0.28 & 0.37 & 0.32 \\
\hline 32 & KS-9503 & 4.2 & 4.9 & 4.6 & 144.0 & 158.5 & 151.3 & 595.4 & 782.0 & 688.7 & 0.29 & 0.43 & 0.36 \\
\hline 33 & KS-8805 & 4.0 & 4.9 & 4.4 & 167.4 & 186.9 & 177.1 & 632.3 & 911.8 & 772.0 & 0.41 & 0.55 & 0.48 \\
\hline 34 & KS-8105 & 3.1 & 4.2 & 3.7 & 157.4 & 178.4 & 167.9 & 494.8 & 743.1 & 618.9 & 0.35 & 0.55 & 0.45 \\
\hline 35 & KS-9010 & 5.7 & 6.7 & 6.2 & 109.8 & 128.3 & 119.1 & 636.9 & 850.9 & 743.9 & 0.39 & 0.55 & 0.47 \\
\hline 36 & KS-9504 & 6.5 & 8.8 & 7.6 & 93.2 & 102.2 & 97.7 & 589.9 & 899.5 & 744.7 & 0.46 & 0.60 & 0.53 \\
\hline 37 & KS-8103 & 19.8 & 7.2 & 13.5 & 45.5 & 60.5 & 53.0 & 905.0 & 428.4 & 666.7 & 0.50 & 0.42 & 0.46 \\
\hline 38 & KS-9501 & 5.6 & 6.7 & 6.1 & 131.9 & 153.9 & 142.9 & 700.7 & 1025.8 & 863.3 & 0.35 & 0.51 & 0.43 \\
\hline 39 & Local collection-1 & 2.6 & 5.3 & 3.9 & 183.1 & 205.1 & 194.1 & 465.8 & 1076.8 & 771.3 & 0.22 & 0.49 & 0.35 \\
\hline 40 & Mukta Hasi & 4.0 & 4.6 & 4.3 & 227.0 & 263.0 & 245.0 & 920.9 & 1212.4 & 1066.7 & 0.44 & 0.56 & 0.50 \\
\hline \multirow{2}{*}{\multicolumn{2}{|c|}{ Mean }} & 61.3 & 83.0 & & 8.9 & 13.1 & & 680.0 & 1246.7 & & 0.43 & 0.62 & \\
\hline & & $\mathrm{S}$ & $\mathrm{G}$ & GX S & $\mathrm{S}$ & $\mathrm{G}$ & GXS & $\mathrm{S}$ & $\mathrm{G}$ & GX S & $\mathrm{S}$ & $\mathrm{G}$ & GXS \\
\hline & S.E (m) & 0.5 & 2.4 & 3.4 & 0.2 & 1.0 & 1.5 & 23.7 & 106.2 & 150.1 & 0.01 & 0.03 & 0.05 \\
\hline & C.D. & 1.5 & 6.8 & 9.6 & 0.7 & 2.9 & 4.2 & 67.0 & 299.4 & 423.4 & 0.02 & 0.10 & 0.13 \\
\hline
\end{tabular}


Fig.1 Histograms showing the frequencies of the mean differences for various traits
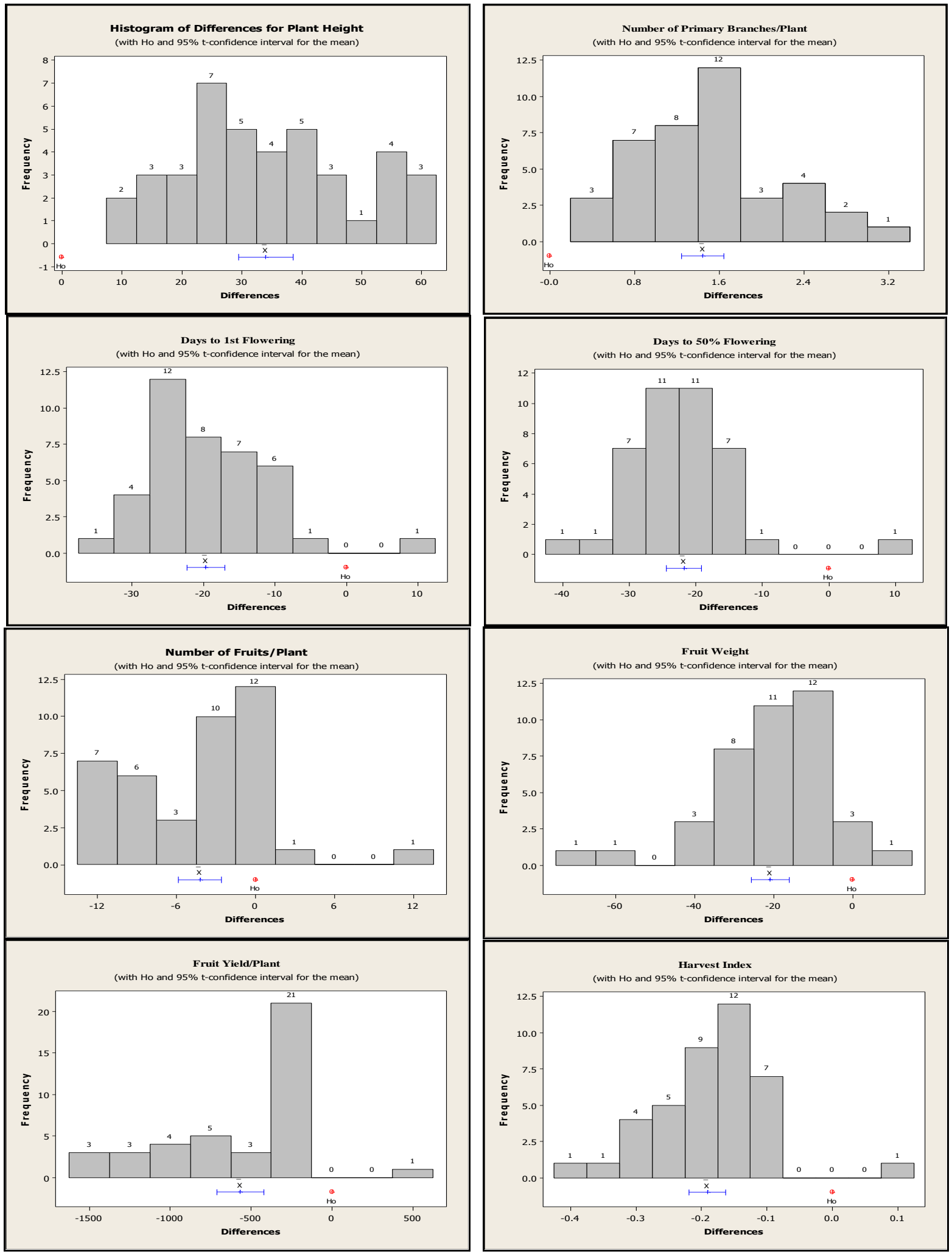
Lower plant height during autumn-winter was due to the prevailing low night temperatures especially in the months of December and January, which partially inhibited the plant growth. Pandit et al., (2010) also found more plant height $(122.39 \mathrm{~cm})$ during springsummer than autumn-winter $(76.59 \mathrm{~cm})$. More number of primary branches per plant was recorded in spring-summer (7.1) than autumn-winter (5.7). During spring-summer they ranged from 5.5 (Mukta Hasi) to 8.3 (Kalo Makra) and during autumn-winter ranged from 4.6 (Mukta Hasi) to 7.0 (Makra Midlong). Significant variation was observed for plant height and number of branches per plant in brinjal previously in various seasons by Singh and Kumar (2005), Kumar et al., (2012), Shinde et al., (2012), Kumar and Arumugam (2013) and Solaimana et al., (2015).

The autumn-winter crop took more days (75.7) for first flowering than spring-summer crop (56). During spring-summer the cultivar Lal Lamba took least number of days for first flowering (39.7) while the cultivar Nandini (63.4) took more number of days for first flowering and the genotype KS-8329 produced first flower in 44.7 days and the genotype KS-2011-1 produced first flower in 88.3 days which was maximum during autumn-winter. Previous studies too indicated that the autumn-winter crop required 71-80 days (Kumar et al., 2012) and 74.8 to 87.9 days (Kumar and Arumugam, 2013) whereas Kharif (Rainy) crop required 36.07 to 49.51 days (Singh and Kumar, 2005) for flowering to be initiated. This could be explained as partial inhibition in vegetative growth during autumn-winter, due to low temperature, delayed the transformation from vegetative to reproductive phase.

Likewise, autumn-winter crop required more days (83) for $50 \%$ flowering where as springsummer crop required less days (61.3) for
$50 \%$ flowering. This indicated the thermosensitive nature of this crop. Lal Lamba took least number of days for $50 \%$ flowering (42.3) and the cultivar Samrat (68.5) took more number of days for $50 \%$ flowering while the genotype KS-8329 produced first flower in 52.7 days and the genotype KS2011-1 produced first flower in 92.7 days which was maximum during autumn-winter. It was understood from the present and previous studies that autumn-winter or rabi sown crops took more days for flowering than spring-summer and rainy or Kharif sown crops.

During spring-summer the number of fruits per plant ranged from 2.1 (KS-2011-1) to 30.4 (Samrat) with a mean value of 8.9 while during autumn-winter it ranged from 2.9 (KS2011-1) to 36.9 (Muktajhuri) with a mean value of 13.1. Similarly Pandit et al., (2010) investigated that lesser number of fruits per plant during spring-summer than autumnwinter. The lesser fruit set in brinjal during spring-summer is attributed to lesser ratio of fertile (long + medium styled) to non-fertile (pseudo short + short) flowers (Shanmugavelu, 1989; Pandit et al., 2010), flower drop and poor fruit set (Baswana et al., 2006) due to environmentally influenced sterility caused by excessive style elongation of two centimetre under high temperature conditions (Pandit et al., 2010). Excessive style elongation causes the pollen grains difficult to reach the stigmatic surface, thereby prevents the pollination which further results in reduced fruit set.

Despite not having significant $G \quad X \quad S$ interaction, the maximum fruit weight was noted during autumn-winter (122.2 g) than spring-summer $(101.4 \mathrm{~g})$. The variety Muktajhuri exhibited lower fruit weight (33.3, $49.4 \mathrm{~g})$ while Muktahasi $(227.0,263.0 \mathrm{~g})$ was having higher fruit weight during both springsummer and autumn-winter. A higher fruit 
yield per plant (1246.7 g) was recorded during autumn-winter than during springsummer $(680.0 \mathrm{~g})$. The cultivar Panna produced higher fruit yield per plant (1392.6 g) and the genotype KS-2011-1 produced lower fruit yield per plant (196.9 g) during spring-summer. The genotype KS-8103 yielded less (428.4 g/plant) and the cultivar Lal Lamba yielded maximum (2575.8 g/plant) during autumn-winter. Prolonged indeterminate growth under warm humid condition and low fruit set as discussed above cumulatively might have decreased both the fruit number and weight, mirroring low fruit yield during spring summer. This was supported by the work of Pandit et al., (2010). Previously several other research workers like Singh and Kumar (2005), Chattopadhyay et al., (2011), Kumar et al., (2012), Shinde et al., (2012), Kumar and Arumugam (2013) and Solaimana et al., (2015) reported significant variation for number of fruits per plant, fruit weight and fruit yield per plant in various seasons.

The harvest index was more during autumnwinter (0.62) than during spring-summer (0.43). This was due to the lower vegetative biomass and the higher fruit yield during autumn-winter than spring-summer. The cultivar Local Collection-1 had lower harvest index (0.2) and the cultivar Samrat was having higher harvest index (0.7) during spring-summer while the genotype KS-8329 had lower harvest index (0.37) and the cultivar Mukta Mala was having higher harvest index (0.85) during autumn-winter. Venkatanaresh et al., (2014) studied and reported high harvest index in brinjal for hybrids.

It is concluded in the present experiment 40 eggplant germplasm was grown during four consecutive seasons, which accounts two spring-summer and two autumn-winter seasons (Table 3). Influence of season on the performance of eggplant was studied and noticed through two factorial ANOVA of Randomized Block Design and paired t-test. Vegetative growth parameters like plant height and number of primary branches per plant were high and earlier flowering took place during spring-summer. Yield parameters like number of fruits per plant, fruit weight, fruit yield per plant and harvest index were recorded high during autumnwinter. But, one genotype KS-8103 had high values for all the yield parameters during spring-summer. Hence, this can be recommended for summer cultivation and for use in further breeding programmes for development high yielding summer varieties. Hence, the thermo-sensitive nature of the eggplant was confirmed.

\section{Acknowledgement}

The first author is highly thankful to the Department of Science and Technology, Government of India for financial assistance through INSPIRE fellowship and to the Director of Research, CSAUA\&T, Kanpur for supplying the seeds of germplasm.

\section{References}

Baswana, K.S., Dahiya, M.S., Kalloo, G., Sharma, N.K., Dhankhar, B.S. and Dudi, B.S. 2006. Brinjal HLB-25: a high temperature tolerant variety. Haryana J. Hort. Sci., 35(3/4): 318319.

Chattopadhyay, A., Dutta, S. and Hazra, P. 2011. Characterization of genetic resources and identification of selection indices of brinjal (Solanum Melongena L.) grown in eastern India. Vegetable Crops Res. Bull., 74: 39-49.

Kowalski, R., Kowalski. G. and Wiercinski, J. 2003. Chemical composition of fruits of three eggplant (Solanum melongena L.) cultivars. Folia Hort., 15(2): 89-95. 
Kumar, S.R. and Arumugam, T. 2013. Variability, heritability and genetic advance for fruit yield, quality and pest and disease incidence in eggplant. Vegetable Sci., 40(1): 111-113.

Kumar, S.R., Arumugam, T. and Premalakshmi, V. 2012. Evaluation and variability studies in local types of brinjal for yield and quality (Solanum melongena L.). Electronic J. Plant Breeding, 3(4): 977-982.

McDonald, J.H. 2014. Handbook of Biological Statistics (3rd ed.), Sparky House Publishing, Baltimore, Maryland, pp 180-185.

Pandit, M.K., Thapa, H., Akhtar, S. and Hazra, P. 2010. Evaluation of brinjal genotypes for growth and reproductive characters with seasonal variation. $J$. Crop and Weed, 6(2): 31-34.

Shanmugavelu, K. G.1989. Production Technology of Vegetable Crops, Oxford and IBH Publishing Co. Ltd., New Delhi, p 276.

Shinde, K.G., Bhalekar, M.N. and Patil, B.T. 2012. Characterization of brinjal (Solanum melongena L.) germplasm. Vegetable Sci., 39(2): 186-188.

Singh, O. and Kumar, J. 2005. Variability, heritability and genetic advance in brinjal. Indian J. Hort., 62(3): 265-267.

Solaimana, A.H.M., Nishizawa, T., Khatun, M. and Ahmad, S. 2015. Physio Morphological Characterization Genetic Variability and Correlation Studies in Brinjal Genotypes of Bangladesh. Computational and Mathematical Biol., 4(1): 1-36.

Venkatanaresh, B., Dubey, A.K., Tiwari, P.K. and Dabbas, M.R. 2014. Line x Tester analysis for yield components and Cercospora leaf spot resistance in brinjal (Solanum melongena L.). Elec. J. Plant Breeding, 5(2): 230-235.

\section{How to cite this article:}

Koundinya, A.V.V., A. Das, P. Pradeep Kumar and Pandit, M.K. 2017. Profiling of Growth and Yield Parameters of Eggplant as Influenced by the Cropping Season. Int.J.Curr.Microbiol.App.Sci. 6(5): 440-448. doi: https://doi.org/10.20546/ijcmas.2017.605.051 